\title{
Filamentary magnetohydrodynamic simulation model, current-vortex method
}

\author{
Yuichi Yatsuyanagia and Toshikazu Ebisuzaki \\ RIKEN (The Institute of Physical and Chemical Research), Wako, Saitama 351-0198, Japan \\ Tadatsugu Hatori \\ Department of Information Science, Kanagawa University, Hiratsuka, Kanagawa 256-1293, Japan \\ Tomokazu Kato \\ Department of Applied Physics, Waseda University, Shinjuku, Tokyo 169-8555, Japan
}

(Received 22 January 2003; accepted 2 June 2003)

\begin{abstract}
A two-dimensional simulation model of the "magnetohydrodynamic (MHD)" vortex method, current-vortex method, is developed. The concept is based on the previously developed current-vortex filament model in three-dimensional space. It is assumed that electric current and vorticity have discontinuous filamentary (point) distributions on the two-dimensional plane, and both the point electric current and the point vortex are confined in a filament. In other words, they share the same point on the two-dimensional plane, which is called the "current-vortex filament." The spatial profiles of the electric current and the vorticity are determined by the sum of such filaments. Time development equations for a filament are obtained by integrating the two-dimensional MHD equations around the filament. It is found that a special-purpose computer, MDGRAPE-2, is capable not only of molecular dynamics simulations but also of MHD simulations, because MDGRAPE-2 accelerates calculations of the Biot-Savart integral. The current-vortex method on MDGRAPE-2 reproduces the result obtained by the traditional MHD code on a general-purpose computer. (1) 2003 American Institute of Physics. [DOI: 10.1063/1.1594725]
\end{abstract}

\section{INTRODUCTION}

The vortex method is one of the most famous techniques in hydrodynamic simulations. ${ }^{1-3}$ This method has some advantages. Kinetic pressure is not necessary for the simulations and can be obtained explicitly from flow velocity afterward if needed. The fine structure of the vorticity can be traced by the method, because a time evolution of the vorticity profile is directly calculated by the vorticity equation, while the vorticity profile is obtained by the rotational differentiation of the velocity field in simulations using the equation of motion.

It is well known that magnetohydrodynamics (MHD) and incompressible hydrodynamics share many features in mathematical formulations, for example,

$$
\begin{aligned}
& \boldsymbol{\nabla} \cdot \boldsymbol{B}=0, \quad \boldsymbol{\nabla} \cdot \boldsymbol{u}=0, \\
& \boldsymbol{\nabla} \times \boldsymbol{B}=\mu_{0} \boldsymbol{j}, \quad \boldsymbol{\nabla} \times \boldsymbol{u}=\boldsymbol{\omega},
\end{aligned}
$$

where $\boldsymbol{B}, \boldsymbol{u}, \boldsymbol{j}$, and $\boldsymbol{\omega}$ are magnetic field, velocity field, electric current density, and vorticity, respectively. Thus, a lot of research effort has been devoted to develop a model where electric current and vorticity coexist. The first current-vortex model for two-dimensional MHD was presented by Fyfe et $a l^{4.5}$ The filamentary MHD model in terms of the Elsässer variables was presented by Kinney et al. ${ }^{6}$ On the other hand, the current-vortex filament model based on the two-fluid equations was developed by Lakhin et al. ${ }^{7}$ However, a MHD

\footnotetext{
${ }^{a)}$ Electronic mail: yyanagi@postman.riken.go.jp
}

model where the vortex method is applied straightforwardly has not been presented. Thus we extend the vortex method to the two-dimensional MHD model, which we call the "current-vortex method."

In the current-vortex method, we assume that the electric current and the vorticity have discontinuous filamentary (point) distributions on the two-dimensional plane, and a current-vortex filament contains both the point electric current and the point vortex. The spatial profiles of the electric current and the vorticity are determined by the sum of the point electric currents and the point vortices in the currentvortex filaments, respectively. Magnetic and velocity fields are obtained by the Biot-Savart integrals. It is remarkable that simulations under high magnetic Reynolds number can be carried out stably by the current-vortex method, even-if the electric resistivity equals zero, because the profiles of the electric current and the vorticity are obtained without the differentiation, and spatial meshes are not necessary to trace the motions of the current-vortex filaments.

However, one must notice the calculation cost of the Biot-Savart integral. It is proportional to $N^{2}$, where $N$ is the number of points on which the electric current and the vorticity exist. It is likely that simulation time is dominated by calculation time for the Biot-Savart integral. Thus, fast calculations of the Biot-Savart integral successfully yield short simulation time. To improve the calculation performance of the Biot-Savart integral, we use MDGRAPE- $2 .{ }^{8}$ It is a special-purpose computer for classical molecular dynamics simulations. It originates from the GRAPE (GRAvity PipE) system. ${ }^{9,10}$ MDGRAPE-2 accelerates calculations of non- 
bonding forces, i.e., Coulomb force, van der Waals force and so on. Rewriting the Biot-Savart integral, one finds that MDGRAPE-2 can calculate the Biot-Savart integral.

The paper is organized as follows. In Sec. II, we describe the basic equations. Basic physical quantities are rewritten in two-dimensional filamentary representations. In Sec. III, we derive equations for filamentary MHD simulations. In Sec. IV, we present our simulation method, and compare the result obtained by the current-vortex method on MDGRAPE-2 with the one obtained by the ordinary MHD code on the general-purpose computer. In Sec. V, we give discussions and conclusions.

\section{BASIC EQUATIONS}

We use the two-dimensional ideal MHD equations

$$
\begin{aligned}
& \frac{\partial \omega_{z}}{\partial t}=-(\boldsymbol{u} \cdot \nabla) \omega_{z}+(\boldsymbol{B} \cdot \boldsymbol{\nabla}) j_{z}, \\
& \frac{\partial A_{z}}{\partial t}=-(\boldsymbol{u} \cdot \boldsymbol{\nabla}) A_{z}, \\
& \boldsymbol{\nabla} \cdot \boldsymbol{u}=0, \\
& \boldsymbol{E}+\boldsymbol{u} \times \boldsymbol{B}=0, \\
& \boldsymbol{B}=-\hat{z} \times \boldsymbol{\nabla} A_{z}, \\
& \omega_{z}(\boldsymbol{r}, t)=\hat{z} \cdot \nabla \times \boldsymbol{u}, \\
& j_{z}(\boldsymbol{r}, t)=\frac{\hat{z}}{\mu_{0}} \cdot \boldsymbol{\nabla} \times \boldsymbol{B},
\end{aligned}
$$

where $\boldsymbol{B}$ and $\boldsymbol{u}$ are the magnetic field and the velocity on the $x-y$ plane, $A_{z}, j_{z}$, and $\omega_{z}$ are the $z$ components of the magnetic vector potential, the electric current density, and the vorticity, respectively. The unit vector in $z$ direction is denoted by $\hat{z}$. The mass density is normalized to unity.

We assume that the electric current and the vorticity have discontinuous distributions, and they are confined in each filament coaxially. At first, let us define explicit formulas of the magnetic vector potential $A_{z}(r, t)$ and the stream function $\psi(\boldsymbol{r}, t)$ :

$$
\begin{gathered}
A_{z}(\boldsymbol{r}, t)=\sum_{i} J_{i}(t) G\left(\boldsymbol{r}-\boldsymbol{r}_{i}(t)\right), \\
\psi(\boldsymbol{r}, t)=\sum_{i} \Omega_{i}(t) G\left(\boldsymbol{r}-\boldsymbol{r}_{i}(t)\right),
\end{gathered}
$$

where $\psi(\boldsymbol{r}, t)$ determines the velocity $\boldsymbol{u}(\boldsymbol{r}, t)$ in the following form:

$$
\boldsymbol{u}(\boldsymbol{r}, t)=-\hat{z} \times \nabla \psi(\boldsymbol{r}, t) .
$$

The notations $\boldsymbol{r}_{i}(t), J_{i}(t)$, and $\Omega_{i}(t)$ are the position vector, the total electric current, and the circulation inside the $i$ th current-vortex filament, respectively. The function $G(r)$ is the two-dimensional Green function for the Poisson equation

$$
G(\boldsymbol{r})=-\frac{1}{2 \pi} \ln |\boldsymbol{r}|
$$

The electric current density $j_{z}(\boldsymbol{r}, t)$ and the vorticity $\omega_{z}(\boldsymbol{r}, t)$ are obtained by

$$
\begin{aligned}
& j_{z}(\boldsymbol{r}, t)=-\nabla^{2} A_{z}(\boldsymbol{r}, t)=\sum_{i} J_{i}(t) \delta\left(\boldsymbol{r}-\boldsymbol{r}_{i}(t)\right), \\
& \omega_{z}(\boldsymbol{r}, t)=-\nabla^{2} \psi(\boldsymbol{r}, t)=\sum_{i} \Omega_{i}(t) \delta\left(\boldsymbol{r}-\boldsymbol{r}_{i}(t)\right),
\end{aligned}
$$

where $\delta(\boldsymbol{r})$ is the two-dimensional Dirac delta function and obeys

$$
\nabla^{2} G(\boldsymbol{r})=-\delta(\boldsymbol{r}) .
$$

Equations (12) and (13) directly show that there is a currentvortex filament at $\boldsymbol{r}_{i}(t)$. Magnetic field $\boldsymbol{B}(\boldsymbol{r}, t)$ and velocity field $\boldsymbol{u}(\boldsymbol{r}, t)$ are obtained by

$$
\begin{aligned}
& \boldsymbol{B}(\boldsymbol{r}, t)=-\hat{z} \times \nabla A_{z}(\boldsymbol{r}, t)=\sum_{i} J_{i}(t) \nabla G\left(\boldsymbol{r}-\boldsymbol{r}_{i}(t)\right) \times \hat{z}, \\
& \boldsymbol{u}(\boldsymbol{r}, t)=-\hat{z} \times \nabla \psi(\boldsymbol{r}, t)=\sum_{i} \Omega_{i}(t) \nabla G\left(\boldsymbol{r}-\boldsymbol{r}_{i}(t)\right) \times \hat{z} .
\end{aligned}
$$

The right-hand sides of Eqs. (15) and (16) are the BiotSavart integrals in the discretized form.

At this point, all the basic quantities are explicitly given by the filamentary representations.

\section{FILAMENTARY MAGNETOHYDRODYNAMICS}

In this section, we show the vorticity equation (1) and the magnetic induction equation (2) rewritten by the filamentary representations. From now on, we use a notation $\boldsymbol{r}_{i}$ instead of $\boldsymbol{r}_{i}(t)$ for simplicity.

\section{A. Vorticity equation}

Now we rewrite the vorticity equation (1) in terms of the filamentary representations $(8)-(16)$. One finds that the vorticity equation becomes

$$
\sum_{i} \frac{d \Omega_{i}(t)}{d t} \delta\left(\boldsymbol{r}-\boldsymbol{r}_{i}\right)-\sum_{i} \Omega_{i}(t) \boldsymbol{\nabla} \cdot\left[\boldsymbol{U}_{i} \delta\left(\boldsymbol{r}-\boldsymbol{r}_{i}\right)\right]=0,
$$

where $\boldsymbol{U}_{i}(\boldsymbol{r})$ is defined by

$$
\begin{aligned}
& \boldsymbol{U}_{i}=\frac{d \boldsymbol{r}_{i}}{d t}-\boldsymbol{u}\left(\boldsymbol{r}_{i}, t\right)-\frac{J_{i}(t)}{\Omega_{i}(t)} \boldsymbol{B}\left(\boldsymbol{r}_{i}, t\right), \\
& \boldsymbol{u}\left(\boldsymbol{r}_{i}, t\right)=\sum_{l \neq i} \Omega_{l}(t) \nabla G\left(\boldsymbol{r}_{i}-\boldsymbol{r}_{l}\right) \times \hat{\boldsymbol{z}}, \\
& \boldsymbol{B}\left(\boldsymbol{r}_{i}, t\right)=\sum_{l \neq i} J_{l}(t) \nabla G\left(\boldsymbol{r}_{i}-\boldsymbol{r}_{l}\right) \times \hat{z} .
\end{aligned}
$$


Note that the self-induced field by the $i$ th filament is excluded in $U_{i}$. To solve Eq. (17), we split the equation to the part that consists of even functions and the one of odd functions.

To obtain the solution concerning the specific filament, say $k$ th filament, we integrate Eq. (17) over a circle area whose center and radius are $\boldsymbol{r}_{k}$ and $\epsilon$, respectively. We assume that the radius $\epsilon$ is small enough and that the distances between the filaments are much larger than $\epsilon$. The integrated equation is given by

$$
\frac{d \Omega_{k}(t)}{d t}-\Omega_{k}(t) \int_{\boldsymbol{r}_{k}} \nabla \cdot\left[\boldsymbol{U}_{k} \delta\left(\boldsymbol{r}-\boldsymbol{r}_{k}\right)\right] d^{2} r=0 .
$$

Here we have used the following relations:

$$
\begin{aligned}
& \int_{\boldsymbol{r}_{k}} \delta\left(\boldsymbol{r}-\boldsymbol{r}_{k}(t)\right) d^{2} r=1, \\
& \int_{\boldsymbol{r}_{k}} \sum_{i} \Omega_{i}(t) \boldsymbol{\nabla} \cdot\left[\boldsymbol{U}_{i} \delta\left(\boldsymbol{r}-\boldsymbol{r}_{i}\right)\right] d^{2} r \\
& =\Omega_{k}(t) \int_{\boldsymbol{r}_{k}} \boldsymbol{\nabla} \cdot\left[\boldsymbol{U}_{k} \delta\left(\boldsymbol{r}-\boldsymbol{r}_{k}\right)\right] d^{2} r .
\end{aligned}
$$

From Eq. (21), the even part solution of Eq. (17) is obtained,

$$
\frac{d \Omega_{k}(t)}{d t}=0,
$$

which gives the conservation of circulation inside the $k$ th filament. The second term in Eq. (21) vanishes because of the symmetry around $\boldsymbol{r}=\boldsymbol{r}_{k}$.

To obtain the odd part solution of Eq. (17), we multiply Eq. (17) by $\left(\boldsymbol{r}-\boldsymbol{r}_{k}\right)$ and get

$$
\begin{aligned}
\sum_{i} & \frac{d \Omega_{i}(t)}{d t}\left(\boldsymbol{r}-\boldsymbol{r}_{k}\right) \delta\left(\boldsymbol{r}-\boldsymbol{r}_{i}\right) \\
& -\sum_{i} \Omega_{i}(t)\left(\boldsymbol{r}-\boldsymbol{r}_{k}\right) \boldsymbol{\nabla} \cdot\left[\boldsymbol{U}_{i} \delta\left(\boldsymbol{r}-\boldsymbol{r}_{i}\right)\right]=0 .
\end{aligned}
$$

Integrating Eq. (25), we obtain

$$
\begin{aligned}
& \frac{d \Omega_{k}(t)}{d t} \int_{r_{k}}\left(\boldsymbol{r}-\boldsymbol{r}_{k}\right) \delta\left(\boldsymbol{r}-\boldsymbol{r}_{k}\right) d^{2} r \\
& \quad-\Omega_{k}(t) \int_{\boldsymbol{r}_{k}}\left(\boldsymbol{r}-\boldsymbol{r}_{k}\right) \boldsymbol{\nabla} \cdot\left[\boldsymbol{U}_{k} \delta\left(\boldsymbol{r}-\boldsymbol{r}_{k}\right)\right] d^{2} r=0 .
\end{aligned}
$$

In this equation, the first term on left-hand side vanishes because of the symmetry around $\boldsymbol{r}=\boldsymbol{r}_{k}$. The remaining term is

$$
\Omega_{k}(t) \int_{\boldsymbol{r}_{k}}\left(\boldsymbol{r}-\boldsymbol{r}_{k}\right) \boldsymbol{\nabla} \cdot\left[\boldsymbol{U}_{k} \delta\left(\boldsymbol{r}-\boldsymbol{r}_{k}\right)\right] d^{2} r=0 .
$$

Thus, the odd part solution of Eq. (17) becomes

$$
\boldsymbol{U}_{k}=0,
$$

or, in the explicit form,

$$
\frac{d \boldsymbol{r}_{k}}{d t}=\boldsymbol{u}\left(\boldsymbol{r}_{k}, t\right)-\frac{J_{k}(t)}{\Omega_{k}(t)} \boldsymbol{B}\left(\boldsymbol{r}_{k}, t\right)
$$

This equation gives the equation of motion of the $k$ th filament. Now we have the solutions of Eq. (17). They are Eqs. (24) and (29).

In our previous work, we obtained the macroscopic force balance equation and macroscopic Faraday's law for the current-vortex filament in three-dimensional configuration correct to the order of $\rho^{-2}$, where $\rho$ is a local radius of curvature of the filament. ${ }^{11}$ If the order is limited to $\rho^{-1}$, the obtained force balance equation is reduced to

$$
\begin{aligned}
\left(\frac{d \boldsymbol{r}}{d t}\right)_{\perp}= & \boldsymbol{u}_{E}(\boldsymbol{r})-\frac{J}{\Omega} \boldsymbol{B}_{\mathrm{E}}(\boldsymbol{r}) \\
& +\frac{\mu_{0} J^{2}}{4 \pi \Omega} \int \frac{\left(\boldsymbol{r}-\boldsymbol{r}^{\prime}\right) \times d \boldsymbol{r}^{\prime}}{\left[\left|\boldsymbol{r}-\boldsymbol{r}^{\prime}\right|^{2}+\alpha^{2}(t) a^{2}(t)\right]^{3 / 2}} \\
& -\frac{\Omega}{4 \pi} \int \frac{\left(\boldsymbol{r}-\boldsymbol{r}^{\prime}\right) \times d \boldsymbol{r}^{\prime}}{\left[\left|\boldsymbol{r}-\boldsymbol{r}^{\prime}\right|^{2}+\beta^{2}(t) a^{2}(t)\right]^{3 / 2}}+O\left(\rho^{-2}\right),
\end{aligned}
$$

where $\boldsymbol{r}$ is a position vector of the filament, $a(t)$ is a radius of the filament, $J$ and $\Omega$ are the total electric current and the circulation in the filament, $\boldsymbol{B}_{E}$ and $\boldsymbol{u}_{E}$ are the external magnetic field and the velocity field which do not include the self-induced field by the filament. The notation $\perp$ denotes the direction perpendicular to the axis of the filament. The parameters $\alpha(t)$ and $\beta(t)$ are the cutoff parameters for the Biot-Savart integral. The details of the cutoff parameter are described in Refs. 11 and 12. Note that the first and second terms on right-hand side in Eq. (30) are $O\left(\rho^{0}\right)$, and the third and the fourth terms are $O\left(\rho^{-1}\right)$. In the two-dimensional case, Eq. (30) is reduced to

$$
\left(\frac{d \boldsymbol{r}}{d t}\right)_{\perp}=\boldsymbol{u}_{\mathrm{E}}(\boldsymbol{r})-\frac{J}{\Omega} \boldsymbol{B}_{\mathrm{E}}(\boldsymbol{r})
$$

because the radius of curvature $\rho$ is infinite in the twodimensional case. Thus the third and fourth terms in Eq. (30) are no more needed, and the self-induced field never affects the self-motion. We see that the Eq. (31) perfectly agrees with Eq. (29).

\section{B. Magnetic induction equation}

In the following, we rewrite the magnetic induction equation (2) in terms of the filamentary representations. One finds that the magnetic induction equation becomes

$$
\begin{aligned}
& \sum_{i} \frac{d J_{i}(t)}{d t} G\left(\boldsymbol{r}-\boldsymbol{r}_{i}\right)-\sum_{i} J_{i}(t) \frac{d \boldsymbol{r}_{i}}{d t} \cdot \nabla G\left(\boldsymbol{r}-\boldsymbol{r}_{i}\right) \\
&=-\sum_{i} J_{i}(t)(\boldsymbol{u} \cdot \boldsymbol{\nabla}) G\left(\boldsymbol{r}-\boldsymbol{r}_{i}\right) .
\end{aligned}
$$

We integrate Eq. (32) over the circle area whose center and radius are $\boldsymbol{r}_{k}$ and $\epsilon$, respectively. In this case also, we assume the parameter $\epsilon$ is small enough. The terms on left-hand side in Eq. (32) are rewritten as 


$$
\begin{aligned}
\int_{\boldsymbol{r}_{k}} \sum_{i} & \frac{d J_{i}(t)}{d t} G\left(\boldsymbol{r}-\boldsymbol{r}_{i}\right) d^{2} r-\int_{\boldsymbol{r}_{k}} \sum_{i} J_{i}(t) \frac{d \boldsymbol{r}_{i}}{d t} \cdot \nabla G\left(\boldsymbol{r}-\boldsymbol{r}_{i}\right) d^{2} r \\
= & \frac{d J_{k}(t)}{d t} \int_{\boldsymbol{r}_{k}} G\left(\boldsymbol{r}-\boldsymbol{r}_{k}\right) d^{2} r+\sum_{i \neq k} \frac{d J_{i}(t)}{d t} \\
& \times \int_{r_{k}} G\left(\boldsymbol{r}-\boldsymbol{r}_{i}\right) d^{2} r \quad-J_{k}(t) \int_{\boldsymbol{r}_{k}} \frac{d \boldsymbol{r}_{k}}{d t} \cdot \nabla G\left(\boldsymbol{r}-\boldsymbol{r}_{k}\right) d^{2} r \\
& -\sum_{i \neq k} J_{i}(t) \int_{\boldsymbol{r}_{k}} \frac{d \boldsymbol{r}_{i}}{d t} \cdot \nabla G\left(\boldsymbol{r}-\boldsymbol{r}_{i}\right) d^{2} r \\
= & -\frac{d J_{k}(t)}{d t} \frac{1}{2} \epsilon^{2}\left(\ln \boldsymbol{\epsilon}-\frac{1}{2}\right)+\sum_{i \neq k} \frac{d J_{i}(t)}{d t} G\left(\boldsymbol{r}_{k}-\boldsymbol{r}_{i}\right) \pi \epsilon^{2} \\
& -\sum_{i \neq k} J_{i}(t) \frac{d \boldsymbol{r}_{i}}{d t} \cdot \boldsymbol{\nabla} G\left(\boldsymbol{r}_{k}-\boldsymbol{r}_{i}\right) \pi \epsilon^{2} .
\end{aligned}
$$

Here we have used the following relations:

$$
\begin{aligned}
& \int_{r_{k}} \nabla G\left(\boldsymbol{r}-\boldsymbol{r}_{k}\right) d^{2} r=0, \\
& \int_{\boldsymbol{r}_{k}} G\left(\boldsymbol{r}-\boldsymbol{r}_{i}\right) d^{2} r=G\left(\boldsymbol{r}_{k}-\boldsymbol{r}_{i}\right) \int_{r_{k}} d^{2} r .
\end{aligned}
$$

The second formula (35) stands on the assumption that the distance $\left|\boldsymbol{r}_{k}-\boldsymbol{r}_{i}\right|$ is large enough, and $G\left(\boldsymbol{r}_{k}-\boldsymbol{r}_{i}\right)$ is approximately constant.

We split the term on right-hand side in Eq. (32) into terms associated with the $k$ th filament and the others. The integrated form is given by

$$
\begin{aligned}
-\int_{r_{k}} \sum_{i} J_{i}(t)(\boldsymbol{u} \cdot \boldsymbol{\nabla}) G\left(\boldsymbol{r}-\boldsymbol{r}_{i}\right) d^{2} r \\
=-J_{k}(t) \int_{\boldsymbol{r}_{k}}(\boldsymbol{u} \cdot \boldsymbol{\nabla}) G\left(\boldsymbol{r}-\boldsymbol{r}_{k}\right) d^{2} r \\
-\sum_{i \neq k} J_{i}(t) \int_{\boldsymbol{r}_{k}}(\boldsymbol{u} \cdot \boldsymbol{\nabla}) G\left(\boldsymbol{r}-\boldsymbol{r}_{i}\right) d^{2} r .
\end{aligned}
$$

We split the velocity field $\boldsymbol{u}$ into two parts,

$$
\begin{aligned}
\boldsymbol{u} & =\sum_{i} \Omega_{i}(t) \boldsymbol{\nabla} G\left(\boldsymbol{r}-\boldsymbol{r}_{i}\right) \times \hat{z} \\
& =\Omega_{k}(t) \boldsymbol{\nabla} G\left(\boldsymbol{r}-\boldsymbol{r}_{k}\right) \times \hat{z}+\boldsymbol{u}\left(\boldsymbol{r}_{k}\right),
\end{aligned}
$$

where $\boldsymbol{u}\left(\boldsymbol{r}_{k}\right)$ is defined by

$$
\boldsymbol{u}\left(\boldsymbol{r}_{k}\right)=\sum_{i \neq k} \Omega_{i}(t) \nabla G\left(\boldsymbol{r}_{k}-\boldsymbol{r}_{i}\right) \times \hat{\boldsymbol{z}},
$$

in the same manner as Eq. (19). Substituting the above expression for $\boldsymbol{u}$ in Eq. (36), we obtain

$$
\begin{aligned}
-J_{k}(t) & \int_{\boldsymbol{r}_{k}(t)}\left\{\left[\Omega_{k}(t) \boldsymbol{\nabla} G\left(\boldsymbol{r}-\boldsymbol{r}_{k}\right) \times \hat{z}+\boldsymbol{u}\left(\boldsymbol{r}_{k}\right)\right] \cdot \nabla\right\} G\left(\boldsymbol{r}-\boldsymbol{r}_{k}\right) d^{2} \boldsymbol{r} \\
- & \sum_{i \neq k} J_{i}(t) \int_{\boldsymbol{r}_{k}(t)}\left\{\left[\Omega_{k}(t) \nabla G\left(\boldsymbol{r}-\boldsymbol{r}_{i}\right) \times \hat{z}+\boldsymbol{u}\left(\boldsymbol{r}_{k}\right)\right] \cdot \boldsymbol{\nabla}\right\} \\
\times & G\left(\boldsymbol{r}-\boldsymbol{r}_{i}\right) d^{2} r \\
= & -J_{k}(t) \int_{\boldsymbol{r}_{k}(t)}\left[\Omega_{k}(t) \nabla G\left(\boldsymbol{r}-\boldsymbol{r}_{k}\right) \times \hat{z}\right] \cdot \nabla G\left(\boldsymbol{r}-\boldsymbol{r}_{k}\right) d^{2} r \\
& -J_{k}(t) \int_{\boldsymbol{r}_{k}(t)} \boldsymbol{u}\left(\boldsymbol{r}_{k}\right) \cdot \boldsymbol{\nabla} G\left(\boldsymbol{r}-\boldsymbol{r}_{k}\right) d^{2} r \\
& -\sum_{i \neq k} J_{i}(t) \int_{\boldsymbol{r}_{k}(t)}\left[\Omega_{k}(t) \nabla G\left(\boldsymbol{r}-\boldsymbol{r}_{i}\right) \times \hat{z}\right] \cdot \nabla G\left(\boldsymbol{r}-\boldsymbol{r}_{i}\right) d^{2} r \\
& -\sum_{i \neq k} J_{i}(t) \int_{\boldsymbol{r}_{k}(t)} \boldsymbol{u}\left(\boldsymbol{r}_{k}\right) \cdot \boldsymbol{\nabla} G\left(\boldsymbol{r}-\boldsymbol{r}_{i}\right) d^{2} r \\
= & -\sum_{i \neq k} J_{i}(t) \boldsymbol{u}\left(\boldsymbol{r}_{k}\right) \cdot \boldsymbol{\nabla} G\left(\boldsymbol{r}_{k}-\boldsymbol{r}_{i}\right) \pi \epsilon^{2} .
\end{aligned}
$$

The relations (34) and (35) have been used here.

From Eqs. (33) and (39), the explicit formula of the magnetic induction Eq. (32) is obtained. Let $\lambda$ be

$$
\lambda=-\left(\ln \epsilon-\frac{1}{2}\right)>0,
$$

and then Eq. (32) becomes

$$
\begin{aligned}
\frac{d J_{k}(t)}{d t}= & -\frac{2 \pi}{\lambda} \sum_{i \neq k} \frac{d J_{i}(t)}{d t} G\left(\boldsymbol{r}_{k}-\boldsymbol{r}_{i}\right)-\frac{2 \pi}{\lambda} \sum_{i \neq k} J_{i}(t) \\
& \times\left[\boldsymbol{u}\left(\boldsymbol{r}_{k}\right)-\frac{d \boldsymbol{r}_{i}}{d t}\right] \cdot \nabla G\left(\boldsymbol{r}_{k}-\boldsymbol{r}_{i}\right) .
\end{aligned}
$$

In the limit of $\epsilon \rightarrow 0$, we obtain

$$
\frac{d J_{k}(t)}{d t}=0 .
$$

This gives the conservation of total electric current inside the $k$ th filament. Now we have a set of equations that determine the motion of the current-vortex filaments. They are given in Eqs. (24), (29), and (42).

\section{SIMULATION METHOD AND RESULTS}

In this section, we give two topics. The first one is a method to calculate the Biot-Savart integral by MDGRAPE-2. The second one is a comparison between the results obtained by the current-vortex method on MDGRAPE- 2 and by the ordinary MHD code on a generalpurpose computer.

\section{A. Biot-Savart integral on MDGRAPE-2}

As is shown in the Introduction, the main purpose of MDGRAPE-2 is to calculate the Coulomb force very fast. MDGRAPE-2 has a capability to calculate the Biot-Savart 
integral also. However, some manipulations of the formulas are required to show how to calculate the Biot-Savart integral by MDGRAPE-2.

The gravitational acceleration at $\boldsymbol{r}$ in three-dimensional space is given by

$$
\boldsymbol{a}(\boldsymbol{r})=-G \sum_{i} m_{i} \frac{\boldsymbol{r}-\boldsymbol{r}_{i}}{\left|\boldsymbol{r}-\boldsymbol{r}_{i}\right|^{3}},
$$

where $r_{i}$ is a position vector of the $i$ th star whose mass is given by $m_{i}$. The summation is performed for all the stars concerned. The calculation of Eq. (43) can be accelerated by MDGRAPE-2.

On the other hand, the magnetic field induced by the electric current filament is obtained by the Biot-Savart integral,

$$
\boldsymbol{B}(\boldsymbol{r})=-\frac{\mu_{0} J}{4 \pi} \int \frac{\left(\boldsymbol{r}-\boldsymbol{r}^{\prime}\right) \times d \boldsymbol{r}^{\prime}}{\left|\boldsymbol{r}-\boldsymbol{r}^{\prime}\right|^{3}},
$$

which gives the magnetic field in three-dimensional space at $\boldsymbol{r}$. The vector $\boldsymbol{r}^{\prime}$ is the position vector of the small element of the filament. The total electric current inside the filament is denoted by $J$. The integral is performed for all the elements $d \boldsymbol{r}^{\prime}$ in the filament. Equation (44) is discretized in the following form:

$$
\boldsymbol{B}(\boldsymbol{r})=-\frac{\mu_{0} J}{4 \pi} \sum_{i} \frac{\left(\boldsymbol{r}-\boldsymbol{r}_{i}^{\prime}\right) \times d \boldsymbol{r}_{i}^{\prime}}{\left|\boldsymbol{r}-\boldsymbol{r}_{i}^{\prime}\right|^{3}}=-\frac{\mu_{0} J}{4 \pi} \sum_{i} \boldsymbol{\xi}_{i} \times d \boldsymbol{r}_{i}^{\prime},
$$

where we introduce the vector $\boldsymbol{\xi}_{i}$ for simplicity, which is defined by

$$
\boldsymbol{\xi}_{i}=\frac{\boldsymbol{r}-\boldsymbol{r}_{i}^{\prime}}{\left|\boldsymbol{r}-\boldsymbol{r}_{i}^{\prime}\right|^{3}}
$$

The components of $\boldsymbol{\xi}_{i}$ and $d \boldsymbol{r}_{i}$ are denoted by

$$
\begin{aligned}
& \boldsymbol{\xi}_{i}=\left(\xi_{i x}, \xi_{i y}, \xi_{i z}\right), \\
& \boldsymbol{d} \boldsymbol{r}_{i}^{\prime}=\left(d r_{i x}^{\prime}, d r_{i y}^{\prime}, d r_{i z}^{\prime}\right) .
\end{aligned}
$$

Incorporating Eqs. (47) and (48) into Eq. (45), the components of Eq. (45) become

$$
\begin{aligned}
& B_{x}(\boldsymbol{r})=-\frac{\mu_{0} J}{4 \pi} \sum_{i}\left(\xi_{i y} d r_{i z}^{\prime}-\xi_{i z} d r_{i y}^{\prime}\right), \\
& B_{y}(\boldsymbol{r})=-\frac{\mu_{0} J}{4 \pi} \sum_{i}\left(\xi_{i z} d r_{i x}^{\prime}-\xi_{i x} d r_{i z}^{\prime}\right), \\
& B_{z}(\boldsymbol{r})=-\frac{\mu_{0} J}{4 \pi} \sum_{i}\left(\xi_{i x} d r_{i y}^{\prime}-\xi_{i y} d r_{i x}^{\prime}\right) .
\end{aligned}
$$

Now, we compare the following formulas that are the components of Eq. (43) with Eqs. (49), (50), and (51):

$$
\begin{aligned}
& a_{x}(\boldsymbol{r})=-\frac{\mu_{0} J}{4 \pi} \sum_{i} \xi_{i x} d r_{i x}^{\prime}, \\
& a_{y}(\boldsymbol{r})=-\frac{\mu_{0} J}{4 \pi} \sum_{i} \xi_{i y} d r_{i x}^{\prime},
\end{aligned}
$$

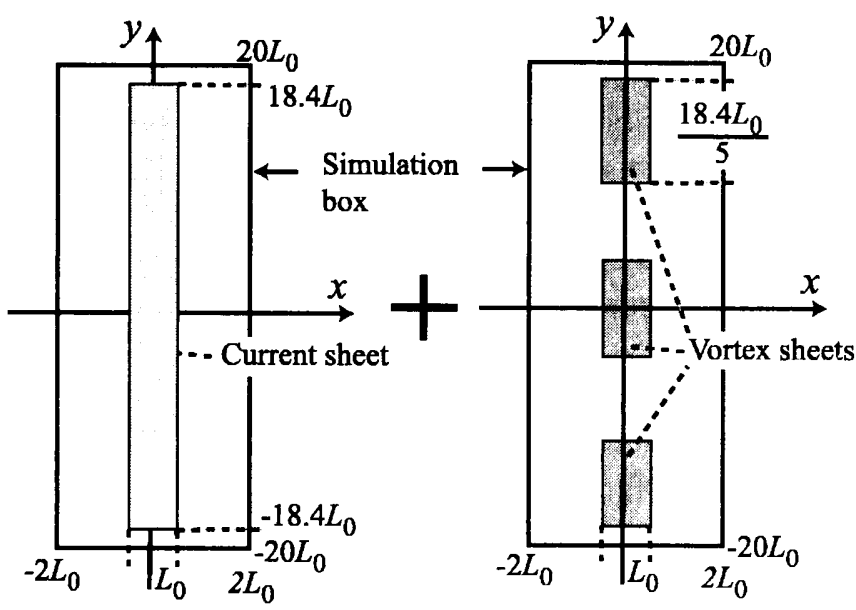

FIG. 1. Initial condition of the simulation is shown.

$$
a_{z}(\boldsymbol{r})=-\frac{\mu_{0} J}{4 \pi} \sum_{i} \xi_{i=} d r_{i x}^{\prime} .
$$

Formulas (52)-(54) are obtained by substituting $\mu_{0} J /(4 \pi)$ for $G$ and $d r_{i x}^{\prime}$ for $m_{i}$ in Eq. (43). Equations (52)-(54) have the forms suitable for MDGRAPE-2. You will find that the components $a_{y}(\boldsymbol{r})$ and $a_{z}(\boldsymbol{r})$ appear in formulas (51) and (50), respectively. All the terms in Eqs. (49)-(51), therefore, can be obtained by substituting $d r_{i, x}^{\prime}, d r_{i v}^{\prime}$, and $d r_{i=}^{\prime}$ for $m_{i}$, respectively. Thus, the Biot-Savart integral (45) can be calculated by MDGRAPE- 2 .

The above-mentioned method is for the threedimensional case. If the two-dimensional problem is considered, the Biot-Savart integral is as follows:

$$
\boldsymbol{B}(\boldsymbol{r})=-\frac{\mu_{0}}{2 \pi} \int \frac{\left(\boldsymbol{r}-\boldsymbol{r}^{\prime}\right) \times j_{z}\left(\boldsymbol{r}^{\prime}\right) \hat{z}}{\left|\boldsymbol{r}-\boldsymbol{r}^{\prime}\right|^{2}} d^{2} r .
$$

MDGRAPE-2 is also able to calculate this kind of integral. The only thing needed is to rewrite the function table in MDGRAPE-2 that determines the formula of the integrand $\xi$ in Eq. (45). For two-dimensional case, we use the following form of $\xi$ :

$$
\xi=\frac{\boldsymbol{r}-\boldsymbol{r}_{i}^{\prime}}{\left|\boldsymbol{r}-\boldsymbol{r}_{i}^{\prime}\right|^{2}}
$$

\section{B. Simulations by MDGRAPE-2 and general-purpose computer}

In this subsection, we show a simulation result obtained by the current-vortex method on MDGRAPE-2, and compare it with the one obtained by the ordinary MHD code on a general-purpose computer. The details of the latter result have been given in Ref. 13. Illustrations of the initial condition are shown in Fig. 1. There are three vortex sheets and an electron current sheet in the system. Initial widths of the current and the vortex sheet are denoted by $L_{0}$. The size of the simulation box is $4 L_{0}$ in the $x$ direction and $40 L_{0}$ in the $y$ direction. Both the current and the vortex sheet have finite lengths in the $y$ direction. The initial length of the current sheet is $36.8 L_{0}$. Initial lengths of the vortex sheets are reduced to $1 / 5$ each, compared with that of current sheet. Ini- 
tial current density and vorticity are given by $j_{0}$ and $\omega_{0}$, respectively, and uniform inside the sheet. We adopt the free boundary condition at all the edges of the simulation box, because the sheets do not deform in $x$ direction near the upper and lower boundaries, if periodic boundary conditions are used. The time scale of simulations is normalized by the Alfvén transit time $\tau_{A}$. The resistive time scale is given by $\tau_{R}=1000 \tau_{A}$.

In the simulations done by MDGRAPE-2, we use the following equations:

$$
\begin{aligned}
& \frac{d \boldsymbol{r}_{k}}{d t}=\boldsymbol{u}\left(\boldsymbol{r}_{k}, t\right)-\frac{J_{k}(t)}{\Omega_{k}(t)} \boldsymbol{B}\left(\boldsymbol{r}_{k}, t\right), \\
& \frac{d J_{k}(t)}{d t}=0, \\
& \frac{d \Omega_{k}(t)}{d t}=0, \\
& \boldsymbol{B}\left(\boldsymbol{r}_{k}, t\right)=\sum_{i \neq k} J_{i}(t) \nabla G\left(\boldsymbol{r}_{k}-\boldsymbol{r}_{i}(t)\right) \times \hat{z}, \\
& \boldsymbol{u}\left(\boldsymbol{r}_{k}, t\right)=\sum_{i \neq k} \Omega_{i}(t) \nabla G\left(\boldsymbol{r}_{k}-\boldsymbol{r}_{i}(t)\right) \times \hat{z} .
\end{aligned}
$$

The last two Eqs. (60) and (61) are calculated by MDGRAPE-2. The magnitudes of the parameters are as follows. The electric current and the circulation of the $i$ th filament are $J_{i}(0)=\Omega_{i}(0)=0.16$. The total number of the current-vortex filaments is $10^{5}$. The magnitudes of the current density and the vorticity depend on the number of the filaments. They are $j_{0}=\omega_{0}=3.4$. Time step is $5.0 \times 10^{-5}$. The number of total time steps calculated is $1.6 \times 10^{3}$. Four MDGRAPE- 2 boards are used in the simulation.

In the simulations done by the general-purpose computer we use the following equations:

$$
\begin{aligned}
& \frac{\partial \omega_{z}}{\partial t}=-(\boldsymbol{u} \cdot \boldsymbol{\nabla}) \omega_{z}+(\boldsymbol{B} \cdot \boldsymbol{\nabla}) j_{z}, \\
& \frac{\partial A_{z}}{\partial t}=-(\boldsymbol{u} \cdot \boldsymbol{\nabla}) A_{z}+\frac{\eta}{\mu_{0}} \nabla^{2} A_{z}, \\
& j_{z}=-\nabla^{2} A_{z}, \\
& \boldsymbol{u}=\int \omega_{z} \hat{z} \times \nabla G\left(\boldsymbol{r}-\boldsymbol{r}^{\prime}\right) d \boldsymbol{r}^{\prime} .
\end{aligned}
$$

The magnitudes of the current density and the vorticity are $j_{0}=\omega_{0}=0.5$. The time step is $2.0 \times 10^{-4}$. Because of the limitation of the simulation code, the electric resistivity $\eta$ is introduced in the simulations on the general-purpose computer. The magnitude of $\eta$ is 0.1 . However, we consider that the resistivity does not alter the result because the time scale concerned is shorter than the resistive time scale. The number of the meshes is $3.3 \times 10^{4}$. We use the FUJITSU VPP700E and the number of processors (PEs) is four. To compare the results obtained by MDGRAPE-2 and the general-purpose computer, we must normalize the typical (a) Electric current

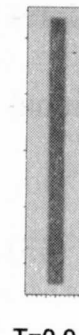

$\mathrm{T}=0.0$
$3.0 \times 10^{2}$

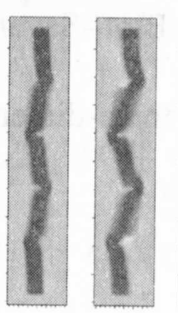

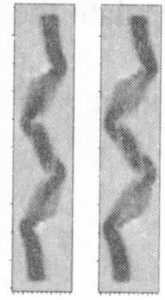

$6.0 \times 10^{2}$ (b) Vorticity

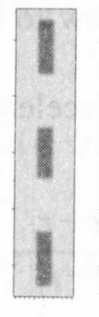

$\mathrm{T}=0.0$

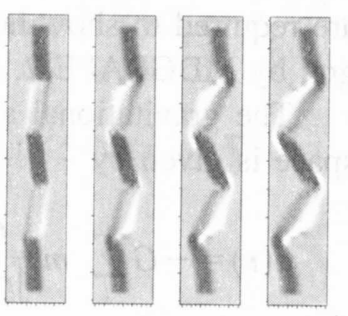

$3.0 \times 10^{2}$

$6.0 \times 10^{2}$
FIG. 2. A simulation result done by the general-purpose computer is shown. The snapshots of the distribution of (a) electric current and (b) vorticity at $T=0.0,1.5 \times 10^{2} \tau_{A}, 3.0 \times 10^{2} \tau_{A}, 4.5 \times 10^{2} \tau_{A}$, and $6.0 \times 10^{2} \tau_{A}$ are given

time scales for both the simulations, because the values of the current density and the vorticity for MDGRAPE-2 are different from those for the general-purpose computer. Let the time scale for the general purpose computer be $T_{0}$. Then the time scale for MDGRAPE-2 $T_{0}^{\prime}$ is given by $T_{0}^{\prime}$ $=T_{0} /\left(1.09 \times 10^{1}\right)$. From now on time scale for MDGRAPE- 2 is normalized by $T_{0}$.

Time evolutions of the current and vortex sheets are shown in Fig. 2. The result shown in Fig. 2 is obtained by the general-purpose computer. In Fig. 2, we can see that the electric current sheet splits into some pieces with time, following the distribution of the vorticity. After $T=1.5$ $\times 10^{2} \tau_{A}$, new configurations appear in both the distributions of the electric current and the vorticity. They exhibit the very similar forms. The electric current and the vorticity evolve to create more overlapping regions where they coexist. The structure is rather filament-like than sheet-like. Once the filaments are formed, they survive stably. This is due to the strong correlation between the electric current and the vorticity. The above comments are for the result obtained by the general-purpose computer. ${ }^{13}$ One finds that basically the same phenomenon can be demonstrated by MDGRAPE-2 that is shown in Fig. 3. The time-evolved distributions of the electric current and the vorticity show very similar results. The calculation time is $110 \mathrm{~min}$ for MDGRAPE-2 and 350 min for the general-purpose computer. Because the number of the filaments for MDGRAPE-2 is larger than the number of the meshes for the general-purpose computer, we cannot compare the calculation time directly. However, it gives evidence that even the MHD simulation using $10^{6}$ filaments (a) Electric current

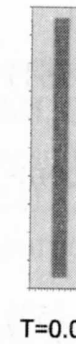

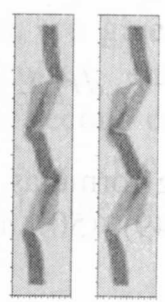

$3.0 \times 10^{2}$
$6.0 \times 10^{2}$ (b) Vorticity

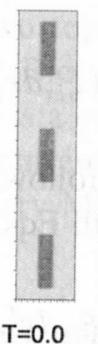

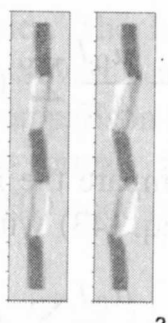

$3.0 \times 10^{2}$

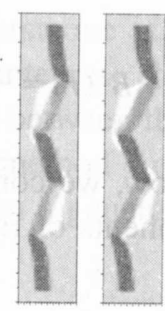

$6.0 \times 10^{2}$
FIG. 3. A simulation result done by MDGRAPE-2 is shown. The snapshots of the distribution of (a) electric current and (b) vorticity at $T=0.0,1.5$ $\times 10^{2} \tau_{A}, 3.0 \times 10^{2} \tau_{A}, 4.5 \times 10^{2} \tau_{A}, 6.0 \times 10^{2} \tau_{A}$ are given. 
can be carried out by MDGRAPE-2 within realistic calculation time.

\section{DISCUSSIONS AND CONCLUSIONS}

We present the simulation model where the electric current and the vorticity are discretized in the same manner as the traditional vortex method in hydrodynamics. The model is suitable for the simulations of the high magnetic Reynolds number. The special-purpose computer, MDGRAPE-2, accelerates the calculation of the Biot-Savart integral. Because the Biot-Savart integral is used in every time step in the current-vortex method, the fast calculation of the BiotSavart integral successfully yields short simulation time. There may be many other applications of MDGRAPE-2.

In this model, we neglect all the viscous parameters, namely, the electric resistivity and the kinetic viscosity. To incorporate these effects, the parameter $\epsilon$ should be finite. However, if $\epsilon$ is finite and constant, a problem arises that the electric current and the vorticity in all the filaments dump at the same rate. Thus we must develop the method to determine $\epsilon$ for each filament appropriately. The other viscous effect may arise from the interactions between many fila- ments. This is just like collisions between the particles. To incorporate these kinds of viscous effects, further investigations are needed.

\section{ACKNOWLEDGMENT}

This work was supported by Special Postdoctoral Research Program at RIKEN.

'A. Leonard, J. Comput. Phys. 37, 289 (1980).

${ }^{2} J$ J. P. Christiansen, J. Comput. Phys. 135, 189 (1997).

${ }^{3}$ P. Koumoutsakos, J. Comput. Phys. 138, 821 (1997).

${ }^{4}$ D. Fyfe and D. Montgomery, J. Plasma Phys. 16, 181 (1976).

${ }^{5}$ D. Fyfe, D. Montgomery, and G. Joyce. J. Plasma Phys. 17, 369 (1977).

${ }^{6} \mathrm{R}$. Kinney, T. Tajima, J. C. McWilliams, and N. Petviashvili, Phys. Plasmas 1, 260 (1994).

${ }^{7}$ V. P. Lakhin, T. J. Schep, and E. Westerhof, Phys. Plasmas 5, 3833 (1998).

${ }^{8}$ T. Narumi, R. Susukita, T. Ebisuzaki, G. McNiven, and B. Elmegreen, Mol. Simul. 21, 401 (1999).

${ }^{9} \mathrm{~J}$. Makino and M. Taiji, Scientific Simulations with Special-Purpose Computers-The GRAPE Systems (Wiley, Chichester, 1998).

${ }^{10}$ T. Ito, J. Makino, T. Ebisuzaki, and D. Sugimoto, Comput. Phys. Commun. 60, 187 (1990).

${ }^{11}$ Y. Yatsuyanagi, T. Hatori, and T. Kato, J. Phys. Soc. Jpn. 65, 745 (1996).

${ }^{12}$ Y. Yatsuyanagi, T. Hatori, and T. Kato, J. Phys. Soc. Jpn. 67, 166 (1998).

${ }^{13}$ Y. Yatsuyanagi, T. Ebisuzaki, T. Hatori, and T. Kato, Phys. Plasmas 9, 446 (2002). 